\title{
Contextual influences on the internal structure of phonetic categories: A distinction between lexical status and speaking rate
}

\author{
J. SEAN ALLEN and JOANNE L. MILLER \\ Northeastern University, Boston, Massachusetts
}

\begin{abstract}
Previous research has shown that phonetic categories have a graded internal structure that is highly dependent on acoustic-phonetic contextual factors, such as speaking rate; these factors alter not only the location of phonetic category boundaries, but also the location of a category's best exemplars. The purpose of the present investigation, which focused on the voiceless category as specified by voice onset time (VOT), was to determine whether a higher order linguistic contextual factor, lexical status, which is known to alter the location of the voiced-voiceless phonetic category boundary, also alters the location of the best exemplars of the voiceless category. The results indicated that lexical status has a more limited and qualitatively different effect on the category's best exemplars than does the acousticphonetic factor of speaking rate. This dissociation is discussed in terms of a production-based account in which perceived best exemplars of a category track contextual variation in speech production.
\end{abstract}

Speech perception is often characterized in terms of a mapping from the acoustic signal onto phonetic categories. A ubiquitous finding in the study of speech perception is that this mapping is highly context dependent. Relevant contextual factors include acoustic-phonetic influences, such as the identity of surrounding segments (e.g., Liberman, Cooper, Shankweiler, \& Studdert-Kennedy, 1967), individual speaker differences (e.g., Nearey, 1989), and speaking rate (e.g., Summerfield, 1981), as well as higher order linguistic influences, such as sentence-level semantic congruity (e.g., Connine, 1987) and lexical status (e.g., Ganong, 1980). In the present paper, we focus on two of these contextual factors: speaking rate, an acoustic-phonetic factor, and lexical status, a higher order linguistic factor. More specifically, we examine whether these two different kinds of contextual factors, which have comparable effects on the location of phonetic category boundaries, can nonetheless be dissociated in their effects on the graded internal structure of phonetic categories.

Consider first the case of speaking rate. In normal speech production, speakers vary their speaking rate considerably (Miller, Grosjean, \& Lomanto, 1984), and the

This research was supported by NIH Grants R01 DC00130 and T32 MH19729 and by a Minority Fellowship from the Acoustical Society of America to the first author. The authors thank Lydia Volaitis and Judith Bürki-Cohen for their contributions to earlier studies that provided the basis for the present paper, Larry Brancazio for his helpful comments on an earlier version of the manuscript, and Elizabeth Dahlager for performing the primary acoustic measurements in Experiment 1. Correspondence concerning this article should be addressed to J. L. Miller, Department of Psychology, Northeastern University, Boston, MA 02115 (e-mail: jlmiller@neu.edu). temporal acoustic properties specifying particular phonetic segments often change in a rate-dependent manner (see Miller, 1981). One such rate-dependent property is voice onset time (VOT), defined acoustically for stop consonants as the time from the onset of the release burst to the onset of periodic energy. VOT differentiates voiced stop consonants (/b d g/), with relatively short VOT values, from voiceless stop consonants (/p t k/), with relatively long VOT values. Summerfield (1975; see also Miller, Green, \& Reeves, 1986; Volaitis \& Miller, 1992) found that VOT varies systematically with speaking rate, so that as speakers slow their speech and produce longer syllables, the VOT interval also becomes longer, especially for voiceless stops. Importantly, listeners are sensitive to this rate dependency and alter the locations of their perceptual phonetic category boundaries accordingly. When listeners are presented with stimuli varying in both VOT and speaking rate and are asked to identify the initial consonant of each stimulus as either a voiced or a voiceless alternative, the voiced-voiceless category boundary shifts toward longer VOT values as speaking rate slows (Summerfield, 1981).

Moreover, the perceptual effect of a change in speaking rate is not confined to the region of the voiced-voiceless category boundary. There is now considerable evidence that phonetic categories have a fine-grained internal category structure, with some members of a category perceived as better exemplars than others (e.g., Kuhl, 1991; Massaro \& Cohen, 1983; Samuel, 1982; Sawusch, 1977). Miller and Volaitis (1989) reported that a change in speaking rate alters this structure, shifting which members of a category are perceived as the best exemplars. Their study focused on the voiceless category. Listeners were presented with two stimulus series varying in VOT, 
one with relatively short syllable durations reflecting a fast speaking rate, and one with relatively long syllable durations reflecting a slower speaking rate. The stimuli within each series ranged from tokens with very short VOT values (heard as /bi/) to tokens with longer VOT values (heard as /pi/) to tokens with VOT values considerably longer than those normally associated with a voiceless stop consonant (heard as a breathy, exaggerated version of $/ \mathrm{pi} /$ ). Listeners were asked to use a numeric scale to rate the category goodness of the initial consonant of each stimulus as $/ \mathrm{p} /$. The resulting response curves for each speaking rate were characterized by a systematic increase in category goodness as VOT increased from the category boundary region toward an optimal range of values, followed by a systematic decrease in category goodness as VOT increased beyond the optimal range. The effect of speaking rate manifested itself as a shift in the goodness function, so that the best exemplars of the /p/ category shifted toward longer VOT values as speaking rate slowed. This basic effect has been replicated many times for the voicing contrast (Volaitis \& Miller, 1992; Wayland, Miller, \& Volaitis, 1994; see also Flege \& Schmidt, 1995) and has been shown to hold as well for the rate-dependent stop-semivowel contrast (Miller, O'Rourke, \& Volaitis, 1997).

Consider now the case of lexical status, a higher order linguistic contextual factor. Lexical status refers to whether or not a given phonetic sequence forms a word in the language. For example, beef is a real word in English, but peef is not. Ganong (1980) created several speech series varying in VOT, such as beef-peef, so that the voiced endpoint of the series formed a real word and the voiceless endpoint formed a nonword. For each series, a phonetically similar, yoked series was created so that the voiced endpoint formed a nonword and the voiceless endpoint formed a word, such as beace-peace. When listeners were asked to identify the initial consonant of these stimuli, Ganong found that the location of the voiced-voiceless category boundary shifted as a function of the lexical status of the endpoint stimuli, so that tokens in the boundary region were more often identified as the alternative consistent with the real word (see also Connine \& Clifton, 1987; Fox, 1984; Miller \& Dexter, 1988; McQueen, 1991; Pitt \& Samuel, 1993). Thus, as in the case of speaking rate, a change in lexical status alters the location of the category boundary.

The main question addressed in the present paper now emerges: Does a change in lexical status, like a change in an acoustic-phonetic contextual factor, such as speaking rate, also affect which stimuli are perceived as the best category exemplars? One possibility is that all contextual factors that shift category boundaries also shift the best exemplars of a category. That is to say, contextual factors operate by shifting the entire category in perceptual space. If this is so, a change in lexical status, like a change in speaking rate, will alter the location of the best exemplars.

Another possibility, however, is that certain contextual factors have a more limited perceptual effect that is confined to the region of the category boundary. In particu- lar, it may be that only those contextual factors that affect a particular acoustic parameter in speech production alter the location of the best exemplars of the corresponding perceptual phonetic category. Recall that in the case of speaking rate, the shift in the location of the best exemplars mirrors the effect of speaking rate in speech production. For example, as speakers slow down their speech, the VOT values associated with a voiceless stop consonant shift toward longer values, just as the best exemplars of the voiceless perceptual category shift toward longer VOT values as speaking rate slows. Thus, the best exemplars of the perceptual category track the acousticphonetic variation associated with speaking rate in speech production. Similar parallels between speech production and the location of the best exemplars of the corresponding perceptual category have also been found for other acoustic-phonetic contextual factors so far examined (Volaitis \& Miller, 1992; Wayland et al., 1994; and see Hodgson \& Miller, 1996, for a similar productionbased best-exemplar shift due to within-category trading relations). These findings are consistent with the view that perceptual shifts in the location of best exemplars arise from a perceptual mechanism that operates so as to track acoustic-phonetic variation in production. On this view, only those contextual factors that alter the critical acoustic parameter in production will alter the location of the best category exemplars along the relevant dimension. As we demonstrate in Experiment 1, a change in lexical status does not alter the VOT values of voiceless stop consonants in speech production. Thus, according to this view, a change in lexical status will not alter the location of the best exemplars of the voiceless category.

To test the two alternatives outlined above, we conducted three experiments. The purpose of Experiment 1 was to demonstrate that lexical status does not systematically alter the VOT values of voiceless stop consonants in speech production. In Experiment 2, a category-goodnessrating paradigm was used to determine whether a change in lexical status alters the location of the best exemplars of the voiceless category. Experiment 3 was a control experiment designed to demonstrate that for our stimuli, speaking rate does alter the location of the best category exemplars, replicating previous findings.

\section{EXPERIMENT 1}

The purpose of Experiment 1 was to demonstrate that VOT does not systematically differ for words and nonwords. Subjects were asked to produce matched monosyllabic words (e.g., peace) and nonwords (e.g., peef) beginning with voiceless stop consonants. The duration of each item was measured to ensure that the words and the nonwords did not differ in overall duration, given that VOT is known to vary with speaking rate (Miller et al., 1986). The critical acoustic measure (given that overall durations were comparable) was the VOT value of each item. In order to ensure that our measure was sufficiently powerful to detect differences in VOT, should they exist, 
Table 1

Word and Nonword Targets Used in Experiment 1

\begin{tabular}{lcccc}
\hline \multicolumn{2}{c}{ Initial Consonant $/ \mathrm{p} /$} & & \multicolumn{2}{c}{ Initial Consonant $/ \mathrm{t} /$} \\
\cline { 5 - 5 } Word & Nonword & & Word & Nonword \\
\hline peace & peef & & test & tesk \\
past & pask & & tarp & tark \\
pave & pathe & & toot & tuke \\
poke & poat & & tube & tude \\
pulp & pulk & & tuft & tust \\
\hline
\end{tabular}

we also included a place of articulation factor in our experimental design, so that half of the stimuli began with the labial consonant $/ \mathrm{p} /$ and half began with the alveolar consonant $/ \mathrm{t} /$. It is well established that /p/ and /t/ differ in VOT, so that syllables beginning with $/ \mathrm{p} /$ have shorter VOT values than do syllables beginning with /t/ (e.g., Lisker \& Abramson, 1964). We therefore expected to find that words and nonwords beginning with /p/ would have significantly shorter VOT values than words and nonwords beginning with /t/.

\section{Method}

Subjects. Six talkers, 3 males and 3 females, participated in the experiment. All were native speakers of American English between 18 and 45 years of age who reported no speech or hearing disorders. The talkers were paid for their participation.

Stimuli. Ten monosyllabic words and 10 matched monosyllabic nonwords served as the target items that were recorded for subsequent acoustic analysis. All the target items began with a voiceless stop consonant: Five of the words and 5 of the nonwords began with $/ \mathrm{p} /$, and the remaining 5 words and 5 nonwords began with $/ \mathrm{t} /$. Each word was matched with a nonword sharing the same initial consonant and vowel, differing only in the place of articulation of the final consonant or consonant cluster (i.e., final-consonant voicing and manner were held constant). The target items are shown in Table $1 .{ }^{1}$

In addition to these 20 target items, 20 filler items were also recorded. The filler items were identical to the target items, except that the initial consonant was voiced instead of voiceless. These filler items were included to prevent any potential artifacts arising from the talkers producing only items with voiceless initial consonants. In each case, the change in initial-consonant voicing altered the lexical status of the item, so that target words (e.g., peace) became nonwords (e.g., beace) and target nonwords (e.g., peef) became words (e.g., beef ). This resulted in 20 voiceless-initial target words and nonwords and 20 voiced-initial filler words and nonwords.

A list was generated with these 40 items as follows. First, eight sublists of 5 items each were created, so that no 2 items from a given matched set (e.g., peef-peace-beef-beace) were in the same or adjacent sublists and so that each sublist contained either two words and three nonwords or three words and two nonwords. These five sublists were then concatenated to form a single block of 40 items. This block was repeated 15 times, each with a different within-sublist randomization, to yield a final list of 600 items.

Procedure. Each talker was recorded individually in a soundtreated booth. The items to be produced were presented to the subject visually on a computer screen, one at a time. Each visual stimulus was displayed for $1,500 \mathrm{msec}$, followed by a pause of $1,200 \mathrm{msec}$ before the next item was displayed. The talkers were instructed to say each item as it appeared on the screen, in a clear but natural manner. Their speech was recorded via a microphone (AKG
C460B) onto digital audiotape (Aiwa HHB 1 PRO DAT recorder). The subjects were given a break midway through the session.

Acoustic analysis. The recording of each target token was transferred to a Pentium PC at a sampling rate of $20 \mathrm{kHz}$, using the CSL system (Kay Elemetrics Corp.). All acoustic measurements were made from the CSL waveform display, with supporting reference to spectrographic analysis and auditory output. VOT was measured from the onset of the initial release burst to the onset of periodicity. Overall duration was measured from the onset of the initial release burst to the offset of energy associated with the final consonant. All reported measurements were made by a single highly trained experimenter. To assess the reliability of these measurements, 2 of the 15 blocks of stimuli from each talker were measured by a second experimenter. Measurements across the two experimenters were highly correlated ( $r=.99$ for VOT and .97 for overall duration) and tightly clustered (a mean difference of less than $1 \mathrm{msec}$ with a standard deviation of $3.85 \mathrm{msec}$ for VOT, and a mean difference of less than $1 \mathrm{msec}$ with a standard deviation of $23.79 \mathrm{msec}$ for overall duration).

\section{Results and Discussion}

Table 2 shows mean VOT and overall duration measurements for the 6 talkers. Two analyses of variance (ANOVAs), one by subjects $\left(F_{1}\right)$ and one by items $\left(F_{2}\right)$, were conducted for both VOT and overall duration. The subjects ANOVAs contained two within-subjects factors, lexical status (word, nonword) and place of articulation (labial, alveolar). The items ANOVAs contained one within-items factor, lexical status (word, nonword), and one between-items factor, place of articulation (labial, alveolar).

Consider first the effects of lexical status and place of articulation on overall duration. There was no reliable difference in overall duration due to lexical status $\left[F_{1}(1,5)=\right.$ $\left.2.45, p>.10 ; F_{2}(1,8)<1\right]$. Although the ANOVA by subjects showed an effect for overall duration due to place of articulation $\left[F_{1}(1,5)=14.76, p<.02\right]$, this difference did not appear in the items ANOVA $\left[F_{2}(1,8)<1\right]$, and on closer inspection of the data, it was discovered that a single outlier stimulus pair (past-pask) was responsible for the inflated duration of the $/ \mathrm{p} /$ tokens relative to the $/ \mathrm{t} /$ tokens. The relatively long durations of the past-pask tokens may be due to a combination of an acoustically long vowel (/æ/) with a long final-consonant cluster (/sk/). A marginally significant interaction between lexical status and place of articulation appeared in the subjects ANOVA $\left[F_{1}(1,5)=4.25, p<.10\right]$, but this interaction did not approach significance in the items ANOVA $\left[F_{2}(1,8)<1\right]$. Taken together, these analyses indicate that neither lexi-

Table 2

Mean Voice Onset Time (VOT) and Overall Duration Measurements (in Milliseconds) for 6 Talkers in Experiment 1

\begin{tabular}{|c|c|c|c|c|c|c|c|c|}
\hline \multirow{3}{*}{$\begin{array}{c}\text { Lexical } \\
\text { Status }\end{array}$} & \multicolumn{4}{|c|}{ Initial Consonant /p/ } & \multicolumn{4}{|c|}{ Initial Consonant/t/ } \\
\hline & \multicolumn{2}{|c|}{ VOT } & \multicolumn{2}{|c|}{ Duration } & \multicolumn{2}{|c|}{ VOT } & \multicolumn{2}{|c|}{ Duration } \\
\hline & $M$ & $S D$ & $M$ & $S D$ & $M$ & $S D$ & $M$ & $S D$ \\
\hline Word & 60 & 15 & 552 & 86 & 72 & 17 & 532 & 80 \\
\hline Nonword & 61 & 13 & 554 & 90 & 73 & 15 & 541 & 83 \\
\hline
\end{tabular}


cal status nor place of articulation systematically influenced the overall duration of the items. ${ }^{2}$

Given that the items were matched for overall duration, consider next the critical question-namely, how lexical status and place of articulation affect VOT. There was no evidence of a difference in VOT due to lexical status $\left[F_{1}(1,5)<1 ; F_{2}(1,8)<1\right]$, but there was strong evidence of a difference in VOT due to place of articulation $\left[F_{1}(1,5)=38.35, p<.01 ; F_{2}(1,8)=12.11, p<.01\right]$. There was no reliable interaction between these two variables $\left[F_{1}(1,5)<1 ; F_{2}(1,8)<1\right]$.

Having established that a change in lexical status does not affect the VOT values of voiceless stop consonants in speech production, we next turned to the primary question of the present investigation-namely, whether a change in lexical status affects the best exemplars of the voiceless category. This question was addressed in Experiment 2 .

\section{EXPERIMENT 2}

The purpose of Experiment 2 was to examine the effect of lexical status on the location of the best exemplars of the /p/ category. The stimuli for the experiment were two matched VOT series, a word-nonword series (beef-peef-*peef) and a nonword-word series (beacepeace-*peace). For each series, VOT ranged from short values appropriate for /b/ to longer VOT values appropriate for /p/ to very long VOT values that led to the perception of a breathy, exaggerated $/ \mathrm{p} /$ (labeled $* / \mathrm{p} /$, for purposes of explication). The subjects were asked to rate the category goodness of the initial consonant of each stimulus as a member of the $/ \mathrm{p} /$ category. On the basis of previous research, it was expected that the change in lexical status would alter the perception of the stimuli in the /b/-/p/ boundary region. The main question addressed in Experiment 2 was whether the change in lexical status would also alter the location of the best exemplars of $/ \mathrm{p} /$. If a contextual factor that affects the boundary also necessarily affects the best exemplars of the category, the location of the best exemplars of /p/ will shift as a function of lexical status. If, on the other hand, only those contextual factors that affect speech production affect the best exemplars of the category, the location of the best exemplars of /p/ will not shift as a function of lexical status.

\section{Method}

Subjects. Twenty subjects participated in the experiment. All the subjects were native speakers of American English between 18 and 45 years of age who reported no speech or hearing disorders and passed a hearing screening test. The subjects were paid for their participation.

Stimuli. The preparation of the stimuli involved five steps, as will be described below. The final product of these five steps was two matched VOT series, a beef-peef-*peef series and a beacepeace-*peace series.

Step 1. A female native speaker of American English produced multiple tokens of /bi/, /bif/ (beef), and /bis/ (beace). Her speech was recorded onto digital audiotape (Aiwa HHB 1 PRO DAT recorder, $\mathrm{AKG} \mathrm{C} 460 \mathrm{~B}$ microphone) in a sound-treated room and were transferred to a Pentium PC at a sampling rate of $20 \mathrm{kHz}$, using the CSL system. One token of the syllable /bi/, which was $343 \mathrm{msec}$ in duration, was selected as the basis for the synthesized series. In anticipation of appending a final fricative to this syllable, the final $96 \mathrm{msec}$ of the vowel were truncated, using the waveform editor in CSL, in order to approximate observed vowel durations for comparable syllables ending in final fricatives (i.e., beef, beace) spoken at a comparable speaking rate by the same talker. This truncation resulted in a /bi/ that was $247 \mathrm{msec}$ in duration. A descending cosine ramp was applied over the final $40 \mathrm{msec}$ of the syllable in order to simulate a realistic amplitude contour for the truncated syllable.

Step 2. Using the ASL program (LPC Parameter Manipulation/ Synthesis Program, Kay Elemetrics), a pitch-synchronous LPC analysis was performed on the waveform of this /bi/, using the autocorrelation method with a filter order of 16 . The results of this analysis were listed in a numeric table where information on peak amplitude, fundamental frequency $(F 0)$, and formant frequencies and bandwidths was available frame by frame. The analyzed data (with the residual excitation) were used to create a synthesized version of the syllable.

Step 3. Using the synthesized syllable as a base, a /bi/-/pi/_*/pi/ series was created. The first stimulus in the series was created by changing the first frame in the synthesized syllable from voiced to voiceless. This was done by changing three parameters in the numeric table and synthesizing a stimulus, using the new values. Specifically, the excitation parameter was changed from the residual to a noise source, the $F 0$ parameter was changed to 0 , and the peak amplitude parameter was scaled down by multiplying the original peak amplitude by 0.2 . The rest of the stimuli within the series were then created by iterating this procedure, incrementing the number of voiceless frames by one on each iteration, until all voiced frames had been changed to voiceless. This resulted in a 49-step series that ranged from a voiced endpoint (VOT $=8 \mathrm{msec}$ ) to an extreme voiceless endpoint in which the entire syllable was voiceless $($ VOT $=247 \mathrm{msec})$. The step size in the series corresponded to the duration of each successive pitch period of the vowel (approximately $5 \mathrm{msec}$ ). Perceptually, the series ranged from a clear $/ \mathrm{bi} / \mathrm{at}$ short VOT values, through a clear /pi/ at intermediate VOT values, to a breathy exaggerated version of /pi/ (*/pi/) at long VOT values. As a result of this procedure, syllable duration $(247 \mathrm{msec})$ was constant across all the tokens in the series (see Allen \& Miller, 1999).

Step 4. Next, the final /f/was excised from a naturally produced utterance of the syllable beef spoken by the same female talker. Similarly, a final /s/, approximately matched for duration to the selected /f/, was excised from the syllable beace spoken by the same talker. With the waveform editor in CSL, the final fricatives were truncated so that each was $216 \mathrm{msec}$ in duration. The final /f/ was appended to each stimulus in the 49-step /bi/-/pi/-*/pi/ series, yielding a 49-step beef-peef-*peef series. Similarly, the final/s/ was appended to each stimulus in the 49-step /bi/-/pi/_*/pi/ series, yielding a 49-step beace-peace-*peace series. The total duration of each stimulus in both series was, thus, $463 \mathrm{msec}$ ( $247 \mathrm{msec}$ plus $216 \mathrm{msec}$ ).

Step 5. A subset of the 98 stimuli (49 beef-peef-*peef tokens and 49 beace-peace-*peace tokens) was selected for presentation to the subjects. The first 20 steps in each series (ranging from VOT = $8 \mathrm{msec}$ to VOT $=97 \mathrm{msec}$ ) and every fifth step thereafter up to a VOT value of $223 \mathrm{msec}$ (VOT = 120, 144, 169, 195, and $223 \mathrm{msec}$ ) were selected, resulting in two 25 -step series. These 50 stimuli were digitally transferred to a different Pentium PC for presentation to the subjects. During presentation, the stimuli were output at a $20-\mathrm{kHz}$ sampling rate and low-pass filtered at $8 \mathrm{kHz}$.

In order to establish the basic lexical status boundary shift for our stimuli, we conducted a preliminary standard two-choice identification experiment. Ten subjects (different from those participating in the main experiment) were presented with 10 repetitions of each of these stimuli, with tokens from the two series randomized 
together. The subjects were asked to identify the initial consonant of each token as either $/ \mathrm{b} /$ or $/ \mathrm{p} /$. The voiced-voiceless category boundary was determined for each subject for each series by fitting a normal ogive to the identification data and calculating the mean of the ogive, which corresponds to the VOT value at which /b/ and $/ \mathrm{p} /$ responses were equally probable. A lexically consistent shift in the boundary location emerged across the two series for each subject, with the beef-peef boundary (mean VOT of $40 \mathrm{msec}$ ) occurring at a longer VOT value than the beace-peace boundary (mean VOT of $34 \mathrm{msec})$. This difference was highly signif icant $[t(9)=$ $6.91, p<.001]$, replicating the basic lexical identification shift for our stimuli. ${ }^{3}$

Procedure. In the main experiment, the subjects were tested in two experimental sessions on separate days. The first experimental session was divided into a familiarization phase, a practice phase, and a test phase. During the familiarization phase, the stimuli from both series (beef-peef-*peef and beace-peace-*peace) were presented in order from shortest to longest VOT values. The order of presentation of the two series was counterbalanced across subjects. The subjects were instructed to listen to the stimuli without making any overt response. They were informed that the initial sound in each series would range from $/ \mathrm{b} /$ to $/ \mathrm{p} /$ to an exaggerated, breathy $/ \mathrm{p} /$. All the subjects reported that they heard this progression. During the practice phase, the subjects heard the 50 tokens from the two series mixed together and presented in random order. The subjects were instructed to listen to each stimulus and rate the goodness of the initial consonant as a member of the /p/ category. They were instructed to press one of seven keys labeled 1 to 7, where 1 corresponded to a very poor exemplar of $/ \mathrm{p} /$ and 7 corresponded to a very good exemplar of $/ \mathrm{p} /$. The subjects were encouraged to use the entire scale from 1 to 7 in making their judgments. The intertrial interval was $2 \mathrm{sec}$, measured from the subject's keypress to the onset of the next stimulus. During the test phase, the subjects heard eight different blocks of the two series. Each block consisted of the 50 tokens from the two series, mixed together and presented in random order. The task was identical to that used during the practice phase. Midway through the test phase, the subjects were given a short break.

The second experimental session was divided into a practice phase and a test phase. The practice phase was identical to the practice phase in the first experimental session, except that the trials were presented in a different random order. The test phase was identical to the test phase in the first experimental session, except that the subjects completed 12 randomized blocks of the two series instead of 8 blocks. Thus, across the two sessions, there were 20 repetitions ( 8 repetitions from the first session and 12 repetitions from the second session) of each of the 50 stimuli, for a total of 1,000 trials in the experiment. The stimuli were presented on line from a Pentium PC binaurally over Sony MDR-V6 headphones at a comfortable listening level that was calibrated identically for all the subjects.

Data analysis. For each stimulus, for each subject, a mean goodness rating across the 20 repetitions of the stimulus was determined. This resulted in two goodness functions for each subject, one for the beef-peef-*peef series and one for the beace-peace-*peace series. Because the individual functions contained local perturbations, we applied a smoothing algorithm to the functions in order to guard against spurious results in the analyses we report below. The smoothed data served as the basis for all reported analyses and figures. The smoothing algorithm was a simple running average of three data points, in which each data point was averaged with the preceding data point and the following data point. The extreme data points (the first and last data points in each function) were not changed. Inspection of these goodness functions indicated that 18 of the 20 subjects yielded the expected nonmonotonic function for each series, with ratings first increasing with an increase in VOT and then decreasing, so that a limited number of stimuli were rated as good category exemplars. The remaining 2 subjects did not show this pattern and were excluded from the analyses reported below. ${ }^{4}$

Following previous work in our laboratory using the goodnessrating paradigm (e.g., Hodgson \& Miller, 1996; Miller \& Volaitis, 1989; Volaitis \& Miller, 1992), we quantified the effect of lexical status on the best exemplars of the category in terms of a bestexemplar range, defined as the range of VOT values for which the ratings were at least $90 \%$ of the maximal rating. The lower and upper limits of the best-exemplar range were calculated for the 18 subjects included in the analyses for both of the smoothed goodness functions in the following manner. First, the maximal rating of each function was determined, and the rating corresponding to $90 \%$ of the maximal value was calculated. The VOT value corresponding to the lower limit of the best-exemplar range was calculated by linear interpolation between the two data points with the lowest VOT values that spanned the $90 \%$ criterion value. The VOT value corresponding to the upper limit of the best-exemplar range was calculated by linear interpolation between the two data points with the highest VOT values that spanned the $90 \%$ criterion value. Although in some previous work (e.g., Volaitis \& Miller, 1992) the location of the maximal rating itself was also employed as a measure of the best-exemplar location, in the present study it was not meaningful to do so, because the individual functions typically lacked a definite peak location but were characterized, instead, by a plateaulike region across the best-exemplar range.

We also examined the effect of lexical status in the region of the category boundary as follows. We selected a criterion value, $60 \%$ of the maximal rating, to serve as a boundary measure. The VOT value determined by the $60 \%$ criterion was calculated by linear interpolation between the two data points with the lowest VOT value that spanned the $60 \%$ criterion value. The $60 \%$ criterion value was selected because, upon inspection of the data, it yielded VOT values in the vicinity of the mean category boundary locations for the two series obtained in the preliminary identification experiment (see the Stimuli section). Thus, the values determined by the $60 \%$ criterion reflect the effect of lexical status in the boundary region.

\section{Results and Discussion}

The mean results for the 18 subjects are shown in the top panel of Figure 1. The figure shows standard nonmonotonic goodness functions, with ratings increasing as VOT increases and then decreasing as VOT values become too long for a good exemplar of $/ \mathrm{p} /$. Overall, the beace-peace-*peace series received higher ratings than the beef-peef-*peef series, giving rise to a global difference in height between the two response curves. This difference in height between the two functions may have occurred because of a tendency for the subjects to rate stimuli more favorably when in a real-word context than when in a nonword context (peace vs. peef). We have also found differences in the relative height of functions in some of our previous studies involving acousticphonetic contextual variables (Hodgson \& Miller, 1996; Volaitis \& Miller, 1992; Wayland et al., 1994), but in each case, as in the present study, the differences in height were not critical to the purpose of the investigation. As was outlined above, our question concerns the locations of the best-exemplar ranges and boundary indices along the VOT continuum. Because these values are calculated separately for each function with respect to the maximal value of that function, the global differences in height do not affect the analyses. 

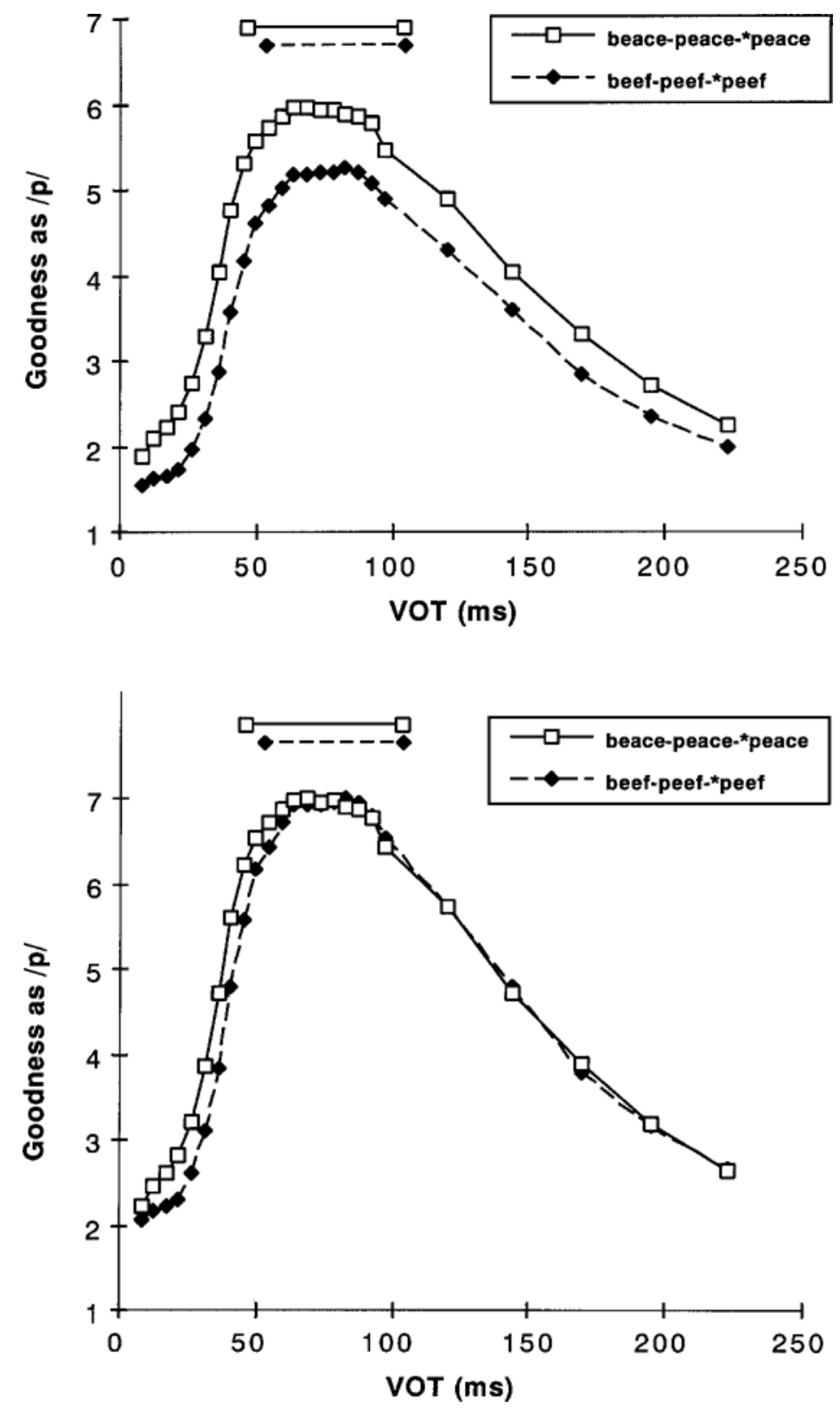

Figure 1. Group goodness ratings as a function of voice onset time (VOT) for the beace-peace-*peace series and the beef-peef-*peef series from Experiment 2 . The range of best exemplars for each function (as defined by the $90 \%$ values listed in Table 2) is indicated by a horizontal bar. Original (smoothed) data are shown in the top panel, and normalized (smoothed) data are shown in the bottom panel.

In order to illustrate the effect of lexical status on the horizontal location of the functions corrected for the difference in height, we normalized the group functions by determining the maximal value in each function and multiplying each data point in the function by a scaling factor so that the new peak of the function would be 7 (the maximal possible value). The normalized group functions for the 18 subjects are shown in the bottom panel of Figure 1. The figure shows that the effect of lexical status emerges as a horizontal shift between the two left edges of the functions (the edges near the category boundary), so that longer VOT values are required to obtain a given rating as $/ \mathrm{p} /$ in the nonword context (peef) than in the word context (peace). Note that this horizontal shift is analogous to the category boundary shift seen with a standard two-choice identification task, in which 
the $/ \mathrm{b} /-/ \mathrm{p} /$ boundary is shifted to longer VOT values when the voiceless endpoint is in a nonword context (beef-peef) than when it is in a word context (beacepeace). Thus, the goodness ratings in the boundary region reflect the expected influence of lexical status on the perception of boundary stimuli. Interestingly, the shift appears throughout the left edge of the functions, with the functions converging near the best-rated exemplars of /p/. At VOT values beyond the best-exemplar region, no systematic difference between the two functions is apparent. Thus, the effect of lexical status, which is clearly evident in the boundary region, seems to disappear within the best-exemplar region.

In order to quantify these observations, the boundary and best-exemplar measures were calculated for each subject for each series as described above. (All calculations were based on the smoothed, nonnormalized data.) The results of these analyses are shown in Table 3. As was predicted, a lexically consistent shift between the two functions was found in the boundary region as defined by the $60 \%$ criterion $[t(17)=5.23, p<.001]$. In addition, a lexically consistent shift was also found at the lower limit of the best-exemplar range defined by the $90 \%$ criterion $[t(17)=5.12, p<.001]$. However, no shift was found at the upper limit of the best-exemplar range defined by the $90 \%$ criterion $[t(17)<1]$. Thus, the effect of lexical status was not confined solely to the boundary region but appeared as well at the lower limit of the bestexemplar range; at the same time, however, lexical status did not shift the entire best-exemplar range. In order to track further the effect of lexical status within the bestexemplar range, we conducted an additional analysis using a more restrictive definition of the best-exemplar range. The best-exemplar ranges for the 18 subjects were recalculated, using the same procedures as before, but with a $95 \%$ criterion instead of a $90 \%$ criterion. The results for the more restrictive $95 \%$ best-exemplar range mirrored those of the less restrictive best-exemplar range and are also shown in Table 3. A lexically consistent shift was found at the lower limit of the best-exemplar range defined by the $95 \%$ criterion $[t(17)=5.17, p<.001]$, but not at the upper limit of the best-exemplar range defined by the $95 \%$ criterion $[t(17)<1]$.

The present results reveal that a change in lexical status systematically alters the category goodness of exem- plars in the region of the category boundary, as was to be expected, but has only a very limited effect on the best exemplars of the category. Specifically, although an effect of lexical status is seen at the lower limit of the bestexemplar range, it disappears within the best-exemplar range, leaving the upper limit unaffected. This pattern is qualitatively different from that seen in previous studies for acoustic-phonetic contextual factors (Miller et al., 1997; Miller \& Volaitis, 1989; Volaitis \& Miller, 1992; Wayland et al., 1994), where the contextual effect shifts the entire best-exemplar range. Thus, lexical status appears to have a qualitatively different, more limited effect on the best exemplars of a category than do acousticphonetic contextual factors.

Before considering the implications of this finding, it is important to rule out the possibility that the failure to find a shift in the entire best-exemplar range in the present study was due not to the contextual factor being lexical status, as we are suggesting, but rather to some (unknown) idiosyncracy of our stimuli. To this end, we conducted a third experiment. The stimuli and procedures for the new experiment were closely patterned after those of the present experiment, except that the contextual factor we tested was speaking rate instead of lexical status. We expected that, unlike the change in lexical status, a change in speaking rate would shift the entire best-exemplar range of the /p/ category for these stimuli.

\section{EXPERIMENT 3}

The purpose of Experiment 3 was to demonstrate that a change in speaking rate, unlike a change in lexical status, would shift the entire best-exemplar range of the $/ \mathrm{p} /$ category for stimuli based on those used in Experiment 2, thus replicating previous findings for the effect of speaking rate on internal category structure (e.g., Miller \& Volaitis, 1989). In Experiment 3, slow and fast versions of the stimuli were presented to the subjects. The beace-peace-*peace series from Experiment 2 served as the slow stimuli, and an edited version of the same series served as the fast stimuli. As in Experiment 2, the subjects were asked to rate the goodness of the initial consonant of each stimulus as a member of the /p/ category. We predicted that a change in speaking rate, unlike

Table 3

Boundary and Best-Exemplar Measures for the

beef-peef-*peef and beace-peace-*peace Goodness Functions, in Milliseconds of Voice Onset Time, in Experiment 2

\begin{tabular}{lccc}
\hline & beef-peef-*peef & beace-peace-*peace & Difference \\
\hline Boundary region & & & \\
60\% boundary measure & 38 & 33 & $5 *$ \\
Best-exemplar ranges & & & \\
$90 \%$ lower limit & 53 & 47 & $6^{*}$ \\
$90 \%$ upper limit & 104 & 103 & 1 (n.s.) \\
$95 \%$ lower limit & 60 & 53 & $7 *$ \\
$95 \%$ upper limit & 94 & 92 & 2 (n.s.) \\
\hline Note-n.s. indicates that $p>.10$. & $* p<.001$. & &
\end{tabular}


the change in lexical status, would shift both the boundary region and the entire best-exemplar range of the goodness function. In terms of the measures used in Experiment 2 , we predicted that a rate-dependent shift between the goodness functions for the slow and the fast series would occur at the boundary as defined by the $60 \%$ criterion, as well as at both the lower and upper limits of the best-exemplar range as defined by both the $90 \%$ and the $95 \%$ criteria.

\section{Method}

Subjects. Twenty new subjects participated in the experiment. All were native speakers of American English between 18 and 45 years of age who reported no speech or hearing disorders and passed a hearing-screening test. The subjects were paid for their participation.

Stimuli. Two series, a slow beace-peace-*peace series and a fast beace-peace-*peace series, served as stimuli in Experiment 3. The 25-step beace-peace-*peace series from Experiment 2 served as the slow series. An edited version of the same series served as the fast series. The series was edited by deleting portions of the vowel and final fricative from each token in the series. Specif ically, the final $87 \mathrm{msec}$ of the vowel were deleted, and a descending cosine ramp was applied over the final $20 \mathrm{msec}$ of the truncated vowel. The new duration of the initial consonant plus vowel was $160 \mathrm{msec}$ (as compared with the original $247 \mathrm{msec}$ ). A 100-msec portion from the middle of the final /s/ was deleted. The new duration of the final/s/ was $116 \mathrm{msec}$ (as compared with the original 216 $\mathrm{msec}$ ). The total duration of each edited token in the fast series was thus $276 \mathrm{msec}$, as compared with a total duration of $463 \mathrm{msec}$ for the slow series. Because the vowel in the fast series was shorter in duration than the vowel in the slow series, there were fewer steps in the fast than in the slow series. Specifically, there were 22 steps in the fast series, as compared with 25 steps in the slow series. The VOT values excluded from the fast series were VOT $=169,195$, and $223 \mathrm{msec}$. All other VOT values were identical in the fast and the slow series.

In order to establish the basic rate-dependent shift in identification for our stimuli, we conducted a preliminary standard twochoice identification experiment. Ten subjects (different from those participating in the main experiment) were presented with 10 repetitions of each of these stimuli, with tokens from the two series randomized together. The subjects were asked to identify the initial consonant of each token as either $/ \mathrm{b} /$ or $/ \mathrm{p} /$. The voiced-voiceless category boundary was determined for each subject for each series by fitting a normal ogive to the identification data and calculating the mean of the ogive, which corresponds to the VOT value at which $/ \mathrm{b} /$ and $/ \mathrm{p} /$ responses were equally probable. A rate-dependent shift in the boundary location emerged across the two series for each subject, with the slow boundary (mean VOT of $36 \mathrm{msec}$ ) occurring at a longer VOT value than the fast boundary (mean VOT of $30 \mathrm{msec}$ ). This difference was highly signif icant $[t(9)=10.70, p<$ $.001]$, replicating the basic rate-dependent identification shift for our stimuli.

Procedure. The procedure for Experiment 3 was identical to that used in Experiment 2, except that there were fewer trials in Experiment 3 because there were fewer stimuli in the fast series relative to the series used in Experiment 2. The subjects were again tested in two experimental sessions. The first experimental session was divided into a familiarization phase, a practice phase, and a test phase. During the familiarization phase, the stimuli from each series were presented in order from shortest to longest VOT values. The order of presentation of the two series was counterbalanced across subjects. During the practice phase, the subjects heard the 47 tokens from the two series mixed together and presented in random order. During the test phase, the subjects heard eight different blocks of the two series. Each block consisted of the 47 tokens from the two series mixed together and presented in random order.

The second experimental session was divided into a practice phase and a test phase. The practice phase was identical to the practice phase in the first experimental session, except that the trials were presented in a different random order. The test phase was identical to the test phase in the first experimental session, except that the subjects completed 12 randomized blocks of the two series, instead of 8 . Thus, across the two sessions, there were 20 repetitions (8 repetitions from the first session and 12 repetitions from the second session) of each of the 47 stimuli, for a total of 940 trials in the experiment.

Data analysis. The data were analyzed in the same manner as in Experiment 2. For each subject, for each series, a smoothed goodness function was derived. Eighteen of the 20 subjects yielded the expected nonmonotonic functions for each series, with ratings first increasing with an increase in VOT and then decreasing, so that a limited number of stimuli were rated as good category exemplars. The remaining 2 subjects did not show this pattern and were excluded from the analyses reported below. ${ }^{5}$ From each smoothed goodness function, the best-exemplar range (using both the $90 \%$ and the $95 \%$ criteria) and the boundary measure (using the $60 \%$ criterion value) were calculated as in Experiment 2.

\section{Results and Discussion}

Mean goodness functions for the 18 subjects are shown in the top panel of Figure 2. Overall, the slow series received somewhat higher goodness ratings, giving rise to a global difference in height between the two response curves. ${ }^{6}$ Following the procedure used in Experiment 2, the group functions were normalized for this difference in height for purposes of illustration, and these normalized functions are shown in the bottom panel of Figure 2. The figure demonstrates that the effect of speaking rate emerges as a comprehensive horizontal shift toward longer VOT values for the slow series than for the fast series, affecting both the boundary region and the entire best-exemplar region of the goodness functions.

In order to quantify these observations, the bestexemplar and boundary measures were calculated for each subject, for each series, as in Experiment 2. The mean results of the boundary analyses and the bestexemplar analyses are shown in Table 4. As was predicted, a rate-dependent shift between the two functions was found in the boundary region as defined by the $60 \%$ criterion $[t(17)=13.22, p<.001]$. Also as was predicted, a rate-dependent shift between the two functions was

Table 4

Boundary and Best-Exemplar Measures for the Slow and Fast beace-peace-*peace Goodness Functions, in Milliseconds of Voice Onset Time, in Experiment 3

\begin{tabular}{lccc}
\hline & Slow & Fast & Difference \\
\hline $\begin{array}{l}\text { Boundary region } \\
\text { 60\% boundary measure }\end{array}$ & 33 & 27 & $6^{*}$ \\
$\begin{array}{l}\text { Best-exemplar ranges } \\
90 \% \text { lower limit }\end{array}$ & 47 & 37 & $10^{*}$ \\
$90 \%$ upper limit & 95 & 78 & $17^{*}$ \\
$95 \%$ lower limit & 54 & 41 & $13^{*}$ \\
$95 \%$ upper limit & 88 & 68 & $20^{*}$ \\
\hline
\end{tabular}

$* p<.001$ 

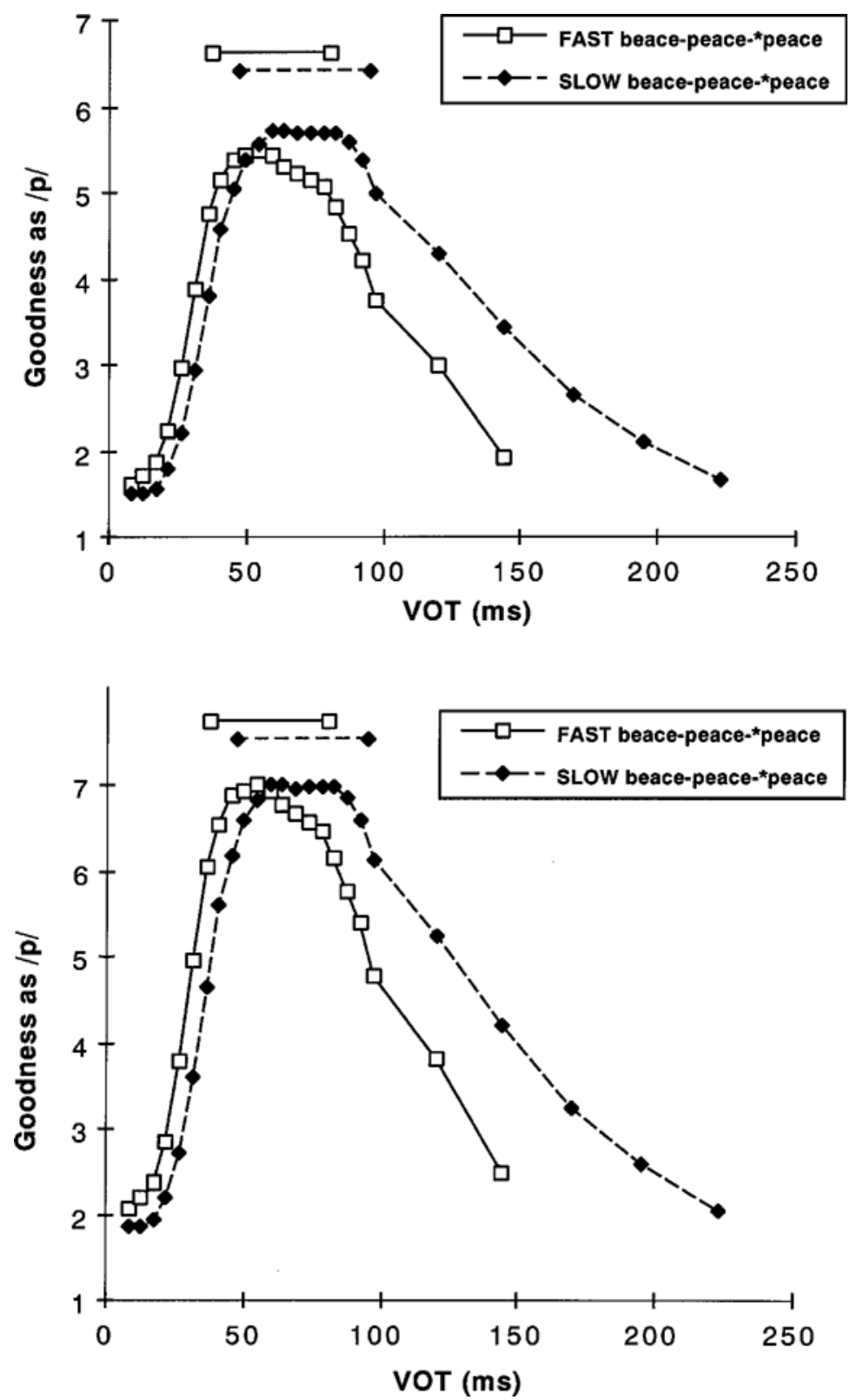

Figure 2. Group goodness ratings as a function of voice onset time (VOT) for the fast and slow beace-peace-*peace series from Experiment 3. The range of best exemplars for each function (as defined by the $90 \%$ values listed in Table 3 ) is indicated by a horizontal bar. Original (smoothed) data are shown in the top panel, and normalized (smoothed) data are shown in the bottom panel.

found at both the lower $[t(17)=7.89, p<.001]$ and the upper $[t(17)=8.81, p<.001]$ limit of the best-exemplar range defined by the $90 \%$ criterion and at both the lower $[t(17)=5.55, p<.001]$ and the upper $[t(17)=10.89, p<$ $.001]$ limit of the best-exemplar range defined by the $95 \%$ criterion.

The present results replicate the findings of Miller and Volaitis (1989), demonstrating that a change in speaking rate from fast to slow shifts the entire best-exemplar range toward longer VOT values. This finding suggests that the failure to find a shift in the upper limit in Experiment 2 was due not to some idiosyncrasy of our stimuli but, rather, to the nature of the contextual factor tested in that study-namely, lexical status. Taken together, the results of Experiments 2 and 3 indicate that the acousticphonetic factor of speaking rate and the higher order lin- 
guistic factor of lexical status have differential effects on the best exemplars of a phonetic category.

\section{GENERAL DISCUSSION}

A pervasive finding in the literature on speech perception is that the mapping between acoustic signal and phonetic category is highly dependent on context, with both acoustic-phonetic factors and higher order linguistic factors systematically altering the location of phonetic category boundaries (see Repp \& Liberman, 1987, for a review). In a recent series of studies, we reported that acoustic-phonetic contextual factors also alter which stimuli within a category are perceived as the best category exemplars (e.g., Miller et al., 1997; Miller \& Volaitis, 1989; Volaitis \& Miller, 1992; Wayland et al., 1994; see also Hodgson \& Miller, 1996). The main objective of the present investigation was to determine whether a higher order linguistic factor, lexical status, which is known to alter category boundary location (Ganong, 1980), also alters the location of the best exemplars of a category.

Two alternatives were outlined in the introduction. The first alternative was that all contextual factors that alter phonetic category boundary locations also alter the location of category best exemplars; in effect, the contextual factor shifts the entire category in perceptual space. The second alternative was that some contextual factors-in particular, higher order linguistic factors that do not affect how the critical phonetic segment is produced-have a more limited effect in perception, operating only within the region of the category boundary.

To investigate these alternatives, we conducted three experiments on the voicing distinction specified by VOT, focusing on the voiceless category. Experiment 1, which was a production study, demonstrated that lexical status does not systematically alter the VOT values of voiceless syllable-initial stop consonants. Experiment 2, which used a category-goodness-rating paradigm, revealed that lexical status had only a limited effect on the location of the perceived best exemplars of the voiceless category. Specifically, a change in lexical status did not shift the entire best-exemplar range of the category (as occurs for acoustic-phonetic factors), but only the lower limit of the range, which borders on the category-boundary region. Experiment 3, which served as a control, showed that the same category-goodness rating paradigm, with variants of the same stimuli, yielded a shift in the entire best-exemplar range when speaking rate, rather than lexical status, was manipulated. Taken together, these results provide support for the second alternative discussed above, with the caveat that an effect for lexical status was found on the lower limit of the best-exemplar range, perhaps as a "spillover" of the boundary effect. Thus, although the effect of lexical status is not confined solely to the boundary region, it is nonetheless qualitatively different and more limited in scope than the effect of acousticphonetic factors.
These findings suggest a dissociation between lower order acoustic-phonetic contextual factors, such as speaking rate, and higher order linguistic contextual factors, such as lexical status, in their effects on the internal structure of phonetic categories. ${ }^{7}$ Although both types of factors systematically alter the location of phonetic category boundaries, they appear to operate differently within the category. As was noted earlier, a number of acoustic-phonetic factors have been shown to shift the entire best-exemplar range of the phonetic category. In contrast, the present study showed that the higher order linguistic factor of lexical status is confined to the category-boundary region and the lower limit of the bestexemplar range, which borders on the boundary region. It appears, then, that the extensively studied categoryboundary shift due to a change in lexical status (see Pitt \& Samuel, 1993) is essentially a boundary phenomenon, as opposed to a comprehensive remapping of the phonetic category in perceptual space, as seen with acousticphonetic contextual factors. This boundary shift has been interpreted in terms of two general classes of models: interactive models (e.g., McClelland \& Elman, 1986), in which higher order representations directly interact with acoustic-phonetic representations during processing via "top-down" feedback, and autonomous models (e.g., Norris, McQueen, \& Cutler, 2000), in which higher order information independently contributes to a separate decision stage of processing. It is unclear at present what predictions these models might make with respect to best-exemplar locations, but any complete model of speech perception must ultimately account for the dissociation we report between an acoustic-phonetic contextual factor and a higher order linguistic contextual factor concerning the location of category best exemplars. ${ }^{8}$

Of course, additional research will be required to determine whether higher order linguistic factors, other than lexical status, that shift category boundary locations, such as sentential-level semantic context (Borsky, Tuller, \& Shapiro, 1998; Connine, 1987; Miller, Green, \& Schermer, 1984), have the same relatively limited effect on the best exemplars of the category. Our interpretation of the dissociation we have found between the effects of acoustic-phonetic factors and lexical status suggests that this dissociation will indeed generalize to other higher order factors, so long as such factors do not affect the relevant acoustic dimension in speech production. This is because our interpretation of this dissociation is based on the differential effects, in general, of acoustic-phonetic factors and higher order linguistic factors in speech production. Acoustic-phonetic factors are those factors that, by definition, manifest themselves in the speech signal. We suggest that the perceptual system tracks the variation in the speech signal associated with acoustic-phonetic factors by shifting the locations of both the boundary and the best exemplars of the appropriate phonetic category. In contrast, higher order linguistic factors are those factors that manifest themselves at a level above that of the speech signal. Assuming that 
these factors do not directly alter the speech signal (as we showed was the case for lexical status in Experiment 1), there is then no associated variation in the speech signal for the perceptual system to track. ${ }^{9}$ Consequently, when the phonetic category boundary shifts as a function of higher order context, the location of the category best exemplars remains stable (or shifts minimally on the boundary side of the best-exemplar range as part of a "spillover" of the boundary effect).

The production-based account described here presumes a close link between the speech perception system and the speech production system, so that the perceptual system tracks acoustic-phonetic variation in production. There are at least two mechanisms by which such a coupling between perception and production could occur. The first possibility is that phonetic perception is carried out via a specialized, articulatory-based speechprocessing module (Liberman \& Mattingly, 1985). The function of this module is to recover the speaker's intended articulatory gestures from the speech stream by reference to richly detailed innate knowledge about the mapping from articulatory gestures to acoustic signal. This knowledge is shared between the speech perception system and the speech production system and results in a perception system that naturally tracks acousticphonetic variation in speech production. The second possible mechanism by which such tracking could occur is one in which listeners store large numbers of specific speech exemplars in memory (e.g., Palmeri, Goldinger, $\&$ Pisoni, 1993). The accumulated experience of listening to distributions of exemplars in different contexts gives rise to a highly sophisticated perceptual system that is finely tuned to acoustic-phonetic contextual variation. Thus, although there is no direct link between the speech perception and the speech production systems, the perceptual system is nonetheless able to track acousticphonetic variation in production by merit of statistical analyses calculated over vast numbers of specific speech exemplars. In either of the two cases just described, acoustic-phonetic tracking is accomplished by altering the mapping between acoustic signal and phonetic categories, shifting both category boundaries and category best exemplars in perceptual space. A higher order contextual factor with no associated acoustic-phonetic variation to track does not give rise to such a comprehensive remapping (although, of course, a higher order contextual effect may arise in the boundary region, as was described above).

We should note that although our findings are consistent with the operation of a perceptual system that actively tracks speech production, there remains an alternative explanation for the observed correspondence between the speech perception and the speech production systems. It has been argued that human languages may have developed in a manner that exploits general auditory principles, so that the acoustic-phonetic form and contextual variation in speech production are selected on the basis of ease of perception (see, e.g., Kluender,
1994). Thus, the correspondence between perception and production may in fact arise from principles of general auditory processing, rather than from specific perceptual mechanisms tracking acoustic-phonetic variation in production. Evidence for such a view comes in part from studies showing that the observed shifts in the location of phonetic category boundaries due to changes in speaking rate can be obtained outside the domain of speech, using nonspeech analogues (e.g., Diehl \& Walsh, 1989; Pastore, Harris, \& Kaplan, 1982; Pisoni, Carrell, $\&$ Gans, 1983). It will be of considerable interest to determine whether comparable shifts due to changes in speaking rate can be obtained for "best exemplars" of these nonspeech analogues. Such a finding would support the view that the sensitivity of best-exemplar location to acoustic-phonetic context also arises from general auditory processes. It may be, however, that even though category boundary locations can be determined (at least in part) by general auditory mechanisms, contextdependent best-exemplar locations arise from speechspecific processes or speech-specific experience (i.e., the two possible mechanisms discussed above). ${ }^{10}$

In summary, we have found a dissociation between a higher order linguistic contextual factor, lexical status, and an acoustic-phonetic factor, speaking rate, in the way in which they influence the best exemplars of perceptual phonetic categories. In line with their differential effects in speech production, a change in speaking rate has a more comprehensive effect on the mapping between speech signal and phonetic category than does a change in lexical status. These findings indicate that not all contex tual factors that influence category boundaries have a strong influence on category "centers" and suggest that, across a range of contextual factors, category "centers" might be more stable than category boundaries. This possibility underscores the importance of considering the internal structure of phonetic categories, in addition to category boundary locations, when developing and testing models of the early stages of spoken language processing.

\section{REFERENCES}

Allen, J. S., \& Miller, J. L. (1999). Effects of syllable-initial voicing and speaking rate on the temporal characteristics of monosyllabic words. Journal of the Acoustical Society of America, 106, 2031-2039.

Borsky, S., Tuller, B., \& Shapiro, L. P. (1998). "How to milk a coat:" The effects of semantic and acoustic information on phoneme categorization. Journal of the Acoustical Society of America, 103, 26702676.

Connine, C. M. (1987). Constraints on interactive processes in auditory word recognition: The role of sentence context. Journal of Memory \& Language, 26, 527-538.

Connine, C. M., \& Clifton, C., JR. (1987). Interactive use of lexical information in speech perception. Journal of Experimental Psychology: Human Perception \& Performance, 13, 291-299.

DieHL, R L., \& WALSH, M. A (1989). An auditory basis for the stimuluslength effect in the perception of stops and glides. Journal of the Acoustical Society of America, 85, 2154-2164.

Flege, J. E., \& Schmidt, A. M. (1995). Native speakers of Spanish show rate-dependent processing of English stop consonants. Phonetica, 52, 90-111. 
Fox, R. A. (1984). Effect of lexical status on phonetic categorization. Journal of Experimental Psychology: Human Perception \& Performance, 10, 526-540.

GANONG, W. F. (1980). Phonetic categorization in auditory word perception. Journal of Experimental Psychology: Human Perception \& Performance, 6, 110-125.

GeFFEN, G., \& Luszcz, M. A. (1983). Are the spoken durations of rare words longer than those of common words? Memory \& Cognition, 11, 13-15.

Hodgson, P., \& Miller, J. L. (1996). Internal structure of phonetic categories: Evidence for within-category trading relations. Journal of the Acoustical Society of America, 100, 565-576.

KLUENDER, K. R. (1994). Speech perception as a tractable problem in cognitive science. In M. A. Gernsbacher (Ed.), Handbook of psycholinguistics (pp. 173-217). San Diego: Academic Press.

KuHL, P. K. (1991). Human adults and human infants show a "perceptual magnet effect" for the prototypes of speech categories, monkeys do not. Perception \& Psychophysics, 50, 93-107.

KuHL, P. K. (2000). Language, mind, and brain: Experience alters perception. In M. S. Gazzaniga (Ed.), The new cognitive neurosciences (pp. 99-115). Cambridge, MA: MIT Press.

Liberman, A. M., Cooper, F. S., Shankweiler, D. P., \& StuddertKennedy, M. (1967). Perception of the speech code. Psychological Review, 74, 431-461.

Liberman, A. M., \& Mattingly, I. G. (1985). The motor theory of speech perception revised. Cognition, 21, 1-36.

LiSKER, L., \& ABRAMSON, A. (1964). A cross-language study of voicing in initial stops: Acoustic measurements. Word, 20, 384-422.

Massaro, D. W., \& Cohen, M. M. (1983). Phonological context in speech perception. Perception \& Psychophysics, 34, 338-348.

McClelland, J. L., \& Elman, J. L. (1986). The TRACE model of speech perception. Cognitive Psychology, 18, 1-86.

McQueEn, J. M. (1991). The influence of the lexicon on phonetic categorization: Stimulus quality in word-final ambiguity. Journal of Experimental Psychology: Human Perception \& Performance, 17, 433 443.

Miller, J. L. (1981). Effects of speaking rate on segmental distinctions. In P. D. Eimas \& J. L. Miller (Eds.), Perspectives on the study of speech (pp. 39-74). Hillsdale, NJ: Erlbaum.

Miller, J. L., \& Dexter, E. R. (1988). Effects of speaking rate and lexical status on phonetic perception. Journal of Experimental Psychology: Human Perception \& Performance, 14, 369-378.

Miller, J. L., \& EimAS, P. D. (1996). Internal structure of voicing categories in early infancy. Perception \& Psychophysics, 58, 1157 1167.

Miller, J. L., Green, K. P., \& Reeves, A. (1986). Speaking rate and segments: A look at the relation between speech production and speech perception for the voicing contrast. Phonetica, 43, 106-115.

Miller, J. L., Green, K. [P.], \& Schermer, T. M. (1984). A distinction between the effects of sentential speaking rate and semantic congruity on word identification. Perception \& Psychophysics, 36, 329-337.

Miller, J. L., Grosiean, F., \& Lomanto, C. (1984). Articulation rate and its variability in spontaneous speech: A reanalysis and some implications. Phonetica, 41, 215-225.

Miller, J. L., O'Rourke, T. B., \& Volaitis, L. E. (1997). Internal structure of phonetic categories: Effects of speaking rate. Phonetica, 54, 121-137.

Miller, J. L., \& Volaitis, L. E. (1989). Effect of speaking rate on the perceptual structure of a phonetic category. Perception \& Psychophysics, 46, 505-512.

Nearey, T. M. (1989). Static, dynamic, and relational properties in vowel perception. Journal of the Acoustical Society of America, 85, 2088-2113.

Norris, D., McQueen, J. M., \& Cutler, A. (2000). Merging information in speech recognition: Feedback is never necessary. Behavioral \& Brain Sciences, 23, 299-370.

Palmeri, T. J., Goldinger, S. D., \& Pisoni, D. B. (1993). Episodic encoding of voice attributes and recognition memory for spoken words. Journal of Experimental Psychology: Learning, Memory, \& Cognition, 19, 309-328.
Pastore, R. E., Harris, L. B., \& Kaplan, J. K. (1982). Temporal order identification: Some parameter dependencies. Journal of the Acoustical Society of America, 71, 430-436.

Pisoni, D. B., Carrell, T. D., \& Gans, S. J. (1983). Perception of the duration of rapid spectrum changes in speech and nonspeech signals. Perception \& Psychophysics, 34, 314-322.

Pitt, M. A., \& Samuel, A G. (1993). An empirical and meta-analytical evaluation of the phoneme identification task. Journal of Experimental Psychology: Human Perception \& Performance, 19, 699-725.

Repp, B. H., \& LibERMAn, A. M. (1987). Phonetic category boundaries are flexible. In S. Harnad (Ed.), Categorical perception: The groundwork of cognition (pp. 89-112). New York: Cambridge University Press.

Samuel, A. G. (1982). Phonetic prototypes. Perception \& Psychophysics, 31, 307-314.

SAWUSCH, J. R. (1977). Peripheral and central processes in selective adaptation of place of articulation in stop consonants. Journal of the Acoustical Society of America, 62, 738-750.

Summerfield, Q. (1975). Aerodynamics versus mechanics in the control of voicing onset in consonant-vowel syllables. In Speech perception (No. 4). Belfast: Queen's University, Department of Psychology.

Summerfield, Q. (1981). Articulatory rate and perceptual constancy in phonetic perception. Journal of Experimental Psychology: Human Perception \& Performance, 7, 1074-1095.

Volaitis, L. E., \& Miller, J. L. (1992). Phonetic prototypes: Influence of place of articulation and speaking rate on the internal structure of voicing categories. Journal of the Acoustical Society of America, 92, 723-735.

Wayland, S. C., Miller, J. L., \& Volaitis, L. E. (1994). The influence of sentential speaking rate on the internal structure of phonetic categories. Journal of the Acoustical Society of America, 95, 2694-2701.

WrIGHT, C. E. (1979). Duration differences between rare and common words and their implications for the interpretation of word frequency effects. Memory \& Cognition, 7, 411-419.

\section{NOTES}

1. Because the items were presented visually to the subjects during the recording session, it was necessary to choose a spelling for the nonwords. We settled on the convention of spelling the nonwords by analogy to their voiced-initial word counterparts. For example, peef was spelled by analogy to beef, poat by analogy to boat, and tuke by analogy to duke.

2. Wright (1979; but see Geffen \& Luszcz, 1983, for counterevidence to this claim) reports that word frequency affects word duration, so that low-frequency words are longer in duration than high-frequency words. To the extent that a nonword can be considered an extremely low frequency word, it might be expected that nonwords would be longer in duration than words. However, we found no evidence of such a difference in the present study.

3. As was expected, the identification functions for both series were highly systematic, with stimuli with short VOT values categorized as /b/ and those with longer VOT values categorized as /p/. Interestingly, however, stimuli with very long VOT values from the beef-peef-*peef series (but not the beace-peace-*peace series) were identified as /b/ rather than $/ \mathrm{p} /$ (i.e., as forming a real word) on a minority of trials by some subjects.

4. Both subjects showed aberrant goodness functions for the beef-peef-*peef series. Specifically, one subject's ratings for this series did not decline to $90 \%$ of the maximal rating at long VOT values, rendering it impossible to compute a best-exemplar range for this series (see the main text for a description of how this range was computed). The other subject's ratings were aberrant in that the goodness function for beef-peef-*peef was very low and broad; the mean peak rating did not exceed 4 , and the width of the best-exemplar range was greater than three standard deviations from the mean width for the group.

5. Both subjects showed aberrant goodness functions for the fast beace-peace-*peace series. Specifically, one subject's ratings for this series did not decline to $90 \%$ of the maximal rating at long VOT values, rendering it impossible to compute a best-exemplar range for this series. 
The other subject's ratings were aberrant in that the goodness function for the series was very low and broad; the mean peak rating did not exceed 4 , and the width of the best-exemplar range was greater than three standard deviations from the mean width for the group.

6. As is apparent from a comparison of Figures 1 and 2, the overall difference in height between the two response curves in Experiment 3 was considerably less than that between the two response curves for Experiment 2 . This might suggest that lexical status and speaking rate have differential effects not only on the horizontal displacement of the functions along the VOT continuum, the main focus of the present investigation, but also on overall ratings. However, as we noted in the Results and Discussion section for Experiment 2, we have found relative height differences in response functions in some of our earlier studies involving acoustic-phonetic variables, and in some cases these differences were as substantial as that found for lexical status in the present paper. For example, a rather large difference in relative height occurred when sentence-level speaking rate was varied, with higher overall ratings given to tokens in fast than in slow sentences (Wayland et al., 1994). Thus, we do not believe it would be appropriate to attribute the relatively large difference in global height in Experiment 2, as compared with the smaller difference in Experiment 3, to a distinction between lexical status and speaking rate, per se.

7. It is of interest to note that the contextual effects of speaking rate and lexical status are also dissociated in another way. In an earlier paper (Miller \& Dexter, 1988), we provided evidence that listeners obligatorily take account of speaking rate (although not necessarily the rate specified by the entire syllable), but not lexical status (see Fox, 1984), when identifying word-initial consonants varying in voicing. Whether this dissociation and the one we report in the present paper arise from a common source remains to be determined.

8. Any complete model of speech perception should also account for any consistent patterns of global differences in the relative height of rating functions for specif ic kinds of contextual variables. For example, if the global difference in height between the two functions in Experiment 2 arises from a tendency to provide higher ratings in the context of a word (peace) than in that of a nonword (peef), a model must account for this difference, as well as elucidate the relation (if any) between this type of "lexical effect" and the effect of lexical status on boundary and best-exemplar locations, the focus of the present paper.

9. Our production-based account does not preclude the possibility that a higher order contextual factor could, in theory, alter the acousticphonetic form of the segment, and in such a case we would predict that the location of the best exemplars of the category would be affected by such a factor, in keeping with the effect of that factor in speech production. Such a case would provide an interesting test of the productionbased account.

10. For a discussion of the ontogeny of internal category structure from various perspectives, see Kluender (1994), Kuhl (2000), and Miller and Eimas (1996).

(Manuscript received February 17, 2000; revision accepted for publication September 20, 2000.) 